\title{
Microsampling and Analysis of Particles from Ancient Mesoamerican Stone Masks Reveal Clues of the Manufacturing Process
}

Timothy R. Rose ${ }^{1}$ and Jane MacLaren Walsh ${ }^{2}$

1. Department of Mineral Sciences, Smithsonian Institution, Washington, D.C., USA

2. Department of Anthropology, Smithsonian Institution, Washington, D.C., USA

We have examined over one hundred ancient stone masks attributed to the Teotihuacan culture, 100 B.C. to 600 A.D. During the examination we were allowed to make high quality silicone molds ostensibly to examine scratches and tool marks left on surfaces and in drill holes of selected masks. When removed from the stone, primarily from deep within drill holes, a few of the molds had tiny grains embedded or adhered to the surface of the silicone. These incidentally collected particles were carefully transferred to standard SEM stubs for study using the Department of Mineral Sciences NovaNano SEM 600 variable pressure scanning electron microscope and ThermoFisher NSS energy dispersive x-ray spectrometer.

Examination of the masks reveals that they are almost entirely of four stone types: travertine, limestone, serpentinite and listwanite. All of these rock types are relatively soft making them easy to carve even with neolithic tools and materials. The masks typically have six of more holes drilled all the way through the edges of the masks or ears and holes drilled into the corners of the eyes and mouths and for nostrils. Although the masks have been cleaned repeatedly, these holes are the primary source of the particles studied.

Some particles appear to be from the same material as the mask. Examples are particles of pyroxene and serpentine minerals from serpentinite and listwanite masks. We interpret these as remnants of the original stone ground into powder during the manufacturing process. However, we found numerous particles of quartz, feldspar and other minerals that cannot be constituent minerals of the masks themselves. These particles could be residue from the soil that the masks were recovered from or abrasive grit used in the manufacturing process. We do not have knowledge of the composition of soils of Teotihuacan. We can surmise, however, that the quartz, feldspars and other minerals found including hornblende, zircon and ilmenite are consistent with an abrasive made from a crushed granitic rock. Quartz and feldspar are considerably harder than all four main stone types from which the masks were made and therefore would have made very effective grinding media.

Cylindrical silica particles typically 5 to 20 microns in diameter were found in 8 of the ten masks examined. Some are tapered and they appear to be solid rods or hollow tubes. Largely featureless on their surfaces, we suspect these are of organic and not mineralogic origin. We have also identified silica diatoms, all of the same type from four different masks, all made from listwanite. The diatoms have been identified as belonging to the freshwater genus Aulacoseira and the cylindrical particles as particles of sponge megasclere (Scott Starratt personal communication, 2013). Particles of this composition and size would make an effective polish for all four mask stone types. Several diatomaceous earth deposits are known in central Mexico and one deposit is composed almost entirely 
of Aulacoseira. In addition, small figurines have been excavated at Teotihuacan that are purportedly made from diatomaceous earth.

Particles of carbonaceous calcium phosphate (apatite) were found from 4 masks. We suspect this is residue from bone used in the manufacturing process or as part of a mixture used as inlay. Titanium oxide, lead carbonate, zinc oxide and barium sulphate, all modern white pigment materials, were found. It is possible that some or all of these are from modern paint that contaminated the objects during exhibition and handling. There is no evidence that the masks were originally painted white.
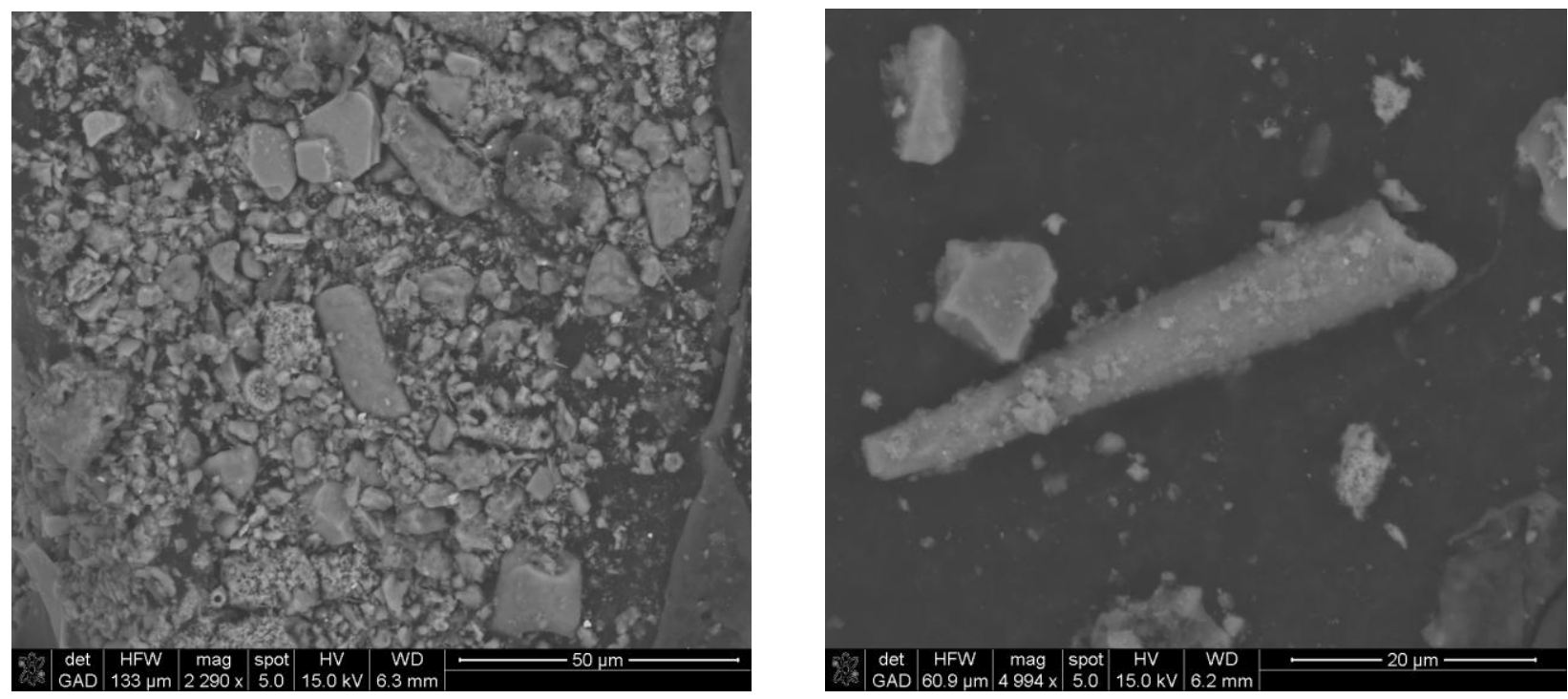

Figure 1. Image on left shows numerous particles removed from a silicone mold taken from a drill hole in a Teotihuacan mask. At right is one of numerous sponge megasclere found on masks of different stone type.
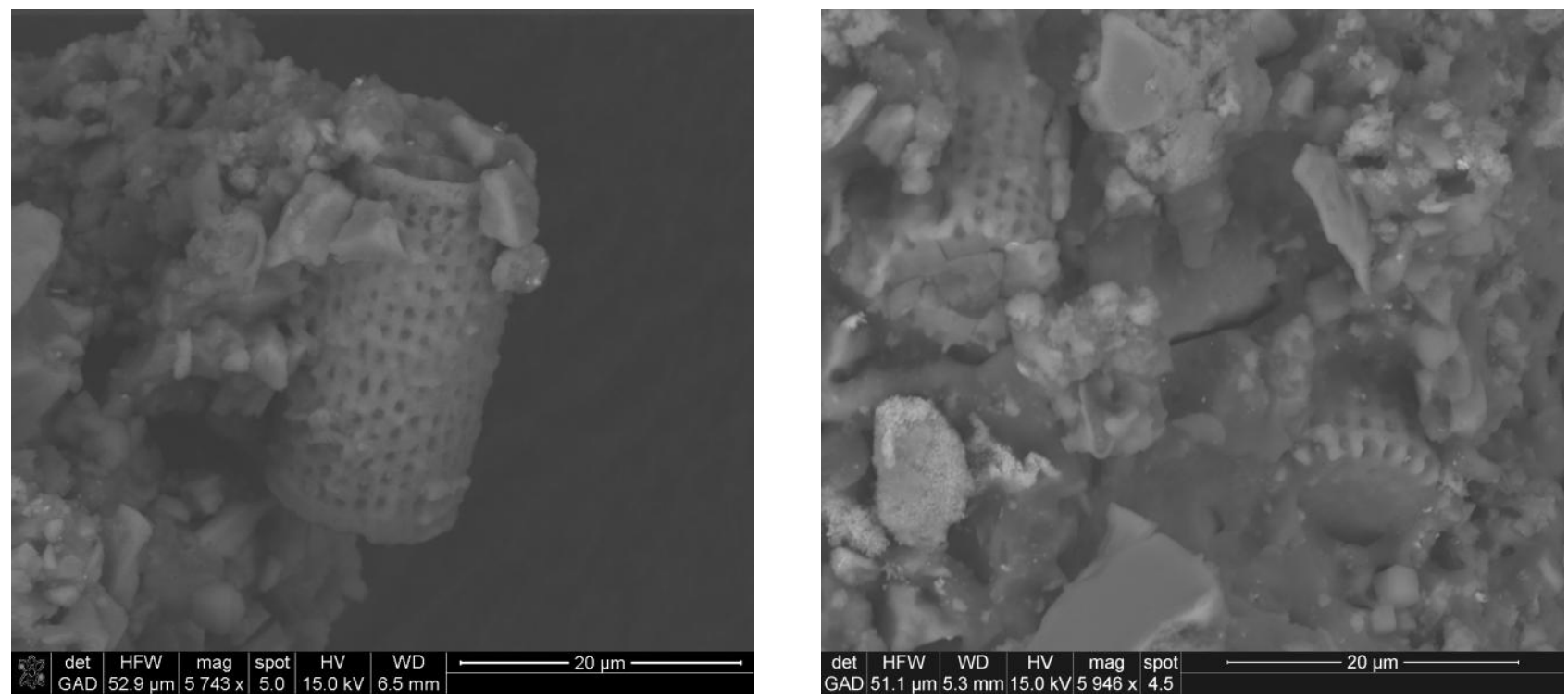

Figure 2. Aulacoseira spp. diatoms found on two listwanite masks from different collections. 